\title{
Compliance with hormonal therapy
}

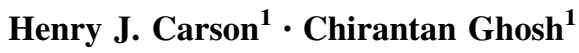

Received: 6 April 2016/Accepted: 19 April 2016/Published online: 26 April 2016

(c) Springer Science+Business Media New York 2016

\section{Dear Sir,}

We read with interest Bradley et al.'s article on financial factors being significant determinants of adherence to hormonal treatment for breast cancer. [1] A number of other reasons can influence adherence to these life-saving medications, [2] so it is useful to address any of these factors that can be managed.

We found that the first step was to assess compliance to hormonal treatment at the time of prescribing, and to follow-up regularly thereafter. Patients in our clinic were contacted to inquire about whether the prescription had been filled and which pharmacy was used. Compliance was screened by then contacting the pharmacy. Problems with filling and using the prescription were discussed with the patients at follow-up visits, including financial barriers to buying the medications. Solutions were provided when possible.

We found that this modest intervention produced substantial results. Compared to a 5-year compliance rate of $49 \%$ in a large integrated managed care setting such as Kaiser Permanente, [3] the compliance rate in our patient group began strong at $96.2 \%$ and increased steadily over a 5-year period to $99.2 \%$. Apparently, the clinic's attentiveness can lead to greater compliance.

And, as Bradley et al. observed, the ability to afford the medications is an important limiting factor in achieving compliance that may be relieved by public policy.

\section{References}

1. Bradley CJ, Dahman B, Jagsi R, Katz S, Hawley S (2015) Prescription drug coverage: implications for hormonal therapy adherence in women diagnosed with breast cancer. Breast Cancer Res Treat 154(2):417-422

2. Reynolds KL, Higgins MJ (2013) Endocrine therapy for breast cancer: a tough pill to swallow. Menopause 20(7):714-716

3. Hershman DL, Kushi LH, Shao T, Buono D, Kershenbaum A, Tsai W-Y, Fehrenbacher L, Gomez SL, Miles S, Neugut AI (2010) Early discontinuation and nonadherence to adjuvant hormonal therapy in a cohort of 8769 early-stage breast cancer patients. J Clin Oncol 28(27):4120-4128
Henry J. Carson

hjcmd@icloud.com

1 The Ghosh Center, Cedar Rapids, IA, USA 